SHORT REPORT

\title{
Sildenafil in neonatal pulmonary hypertension due to impaired alveolarisation and plexiform pulmonary arteriopathy
}

\author{
M Chaudhari, M Vogel, C Wright, J Smith, S G Haworth
}

Arch Dis Child Fetal Neonatal Ed 2005;90:F527-F528. doi: 10.1136/adc.2004.062885

We report a case of severe pulmonary hypertension in a neonate associated with impaired alveolarisation and plexiform pulmonary arteriopathy. Treatment with oral sildenafil in addition to inhaled nitric oxide (NO) resulted in recovery from the pulmonary hypertensive crisis. Long term sildenafil therapy was associated with complete resolution of the pulmonary hypertension.

$\mathrm{D}$ evelopment of the pulmonary vasculature and pulmonary alveolarisation are interdependent in late gestation and early postnatal life. ${ }^{1}$ Impaired alveolarisation is associated with pulmonary vascular disease and pulmonary hypertension. ${ }^{2}$

Inhaled nitric oxide (NO) is currently the gold standard for treatment of severe persistent pulmonary hypertension of the newborn (PPHN). ${ }^{3}$ Extra-corporeal membrane oxygenation (ECMO) is reserved for unresponsive cases with underlying reversible lung pathology. We describe here a neonate with severe pulmonary hypertension in whom the introduction of oral sildenafil in addition to inhaled NO led to control of the pulmonary hypertensive crises. Long term use of sildenafil was associated with resolution of the pulmonary hypertension at the age of 1 year.

\section{CASE REPORT}

A 7 day old female neonate was admitted with respiratory distress, central cyanosis, and floppy episodes. She was born at 38 weeks gestation after induction of delivery. The pregnancy was conceived after clomid therapy and complicated by pregnancy induced hypertension. There was no history of oligohydramnios, premature rupture of the membranes, or meconium stained liquor. At birth she was in good condition (weight $4.4 \mathrm{~kg}$ ) and required no resuscitation.

On admission her heart rate was 160/min and her respiratory rate was $60 / \mathrm{min}$ with moderate sub-costal recession. Systemic saturation was $85 \%$ in air and blood pressure was $86 / 40 \mathrm{~mm} \mathrm{Hg}$. Cardiovascular examination revealed right ventricular hyperactivity, a loud pulmonary component of the second heart sound, a grade 3/6 systolic murmur at the left sternal edge, and normal pulses. The liver edge was palpable $2 \mathrm{~cm}$ below the right costal margin and the chest was clear with good bilateral air entry. An electrocardiogram (ECG) showed evidence of right atrial and right ventricular hypertrophy $(\mathrm{RVH})$. Chest $x$ ray revealed normal cardiac and mediastinal contours and no focal lung lesions. Echocardiogram revealed normal intracardiac anatomy, an enlarged right atrium and right ventricle, a right to left atrial shunt, and moderate tricuspid valve regurgitation (TR). Right ventricular (RV) function was moderately impaired and the TR jet was $5.5 \mathrm{~m} / \mathrm{s}$.

\begin{tabular}{ll} 
Table 1 & Cardiac catheter data $^{*}$ \\
\hline Condition & Observation \\
\hline Weight & $4.2 \mathrm{~kg}$ \\
Height & $52 \mathrm{~cm}$ \\
$\mathrm{BSA}$ & $0.58 \mathrm{~m}^{2}$ \\
$\mathrm{O}_{2}$ saturation & $95 \%$ \\
Haemodynamics & RA (mean): 6 \\
(mm Hg) & LA (mean): 5 \\
& PA: $83 / 45 / 60$ \\
& AO: $63 / 35 / 40$ \\
Vascular & TPR: 16 \\
resistance (units $/ \mathrm{m}^{2}$ ) & PAVR: 14.6 \\
Blood flow & Qp: 3.75 \\
$\left(1 /\right.$ min $\left./ \mathrm{m}^{2}\right)$ & Qs: 2.45 \\
Angiography & Moderate RV dysfunction \\
& Moderate tricuspid regurgitation \\
& Normal intracardiac anatomy \\
\hline
\end{tabular}

${ }^{*}$ General anaesthesia/intermittent positive pressure ventilation: $\mathrm{Fio}_{2}$ 0.65 , NO $20 \mathrm{ppm}$, milrinone $0.75 \mu \mathrm{g} / \mathrm{kg} / \mathrm{min}$.

BSA, body surface area.

The condition of the patient deteriorated rapidly with worsening central cyanosis, hypoxaemia, and acute circulatory collapse secondary to raised pulmonary vascular resistance. She was sedated, paralysed, and ventilated with an $\mathrm{FiO}_{2}$ of 0.8 and inhaled NO (20 ppm). Inotropic support (milrinone) and antibiotics were commenced. Despite these medical manoeuvres, the patient remained in a low cardiac output state with recurrent pulmonary hypertensive crises on handling and endotracheal suction. Investigations revealed a negative sepsis screen and normal renal and liver function. Cardiac catheterisation confirmed supra-systemic pulmonary artery pressure and no structural cardiac abnormality (table 1).

A lung biopsy was performed to exclude primary lung pathology and revealed widespread pulmonary vasculopathy with intimal proliferation and marked medial thickening and severe patchy impaired alveolarisation (fig 1A,B). In view of these abnormalities, the patient was considered unsuitable for ECMO support. Oral sildenafil was introduced on the 4th day after admission at a initial dose of $0.2 \mathrm{mg} / \mathrm{kg} / \mathrm{dose}$ four times a day, increasing to a maximum of $1 \mathrm{mg} / \mathrm{kg} / \mathrm{dose}$ every $4 \mathrm{~h}$. This was associated with control of the pulmonary hypertensive crises and cardiac failure. Inhaled NO was discontinued 2 days later and the patient was weaned off respiratory and inotropic support after 4 days. Oxygen therapy was gradually withdrawn over 2 weeks. The patient

\footnotetext{
Abbreviations: BSA, body surface area; $c$ GMP, cyclic-guanosine 5 monophosphate; ECG, electrocardiogram; ECMO, extra-corporeal membrane oxygenation; NO, nitric oxide; PPHN, persistent pulmonary hypertension of the newborn; RV, right ventricular; $\mathrm{RVH}$, right ventricular hypertrophy; TR, tricuspid valve regurgitation
} 


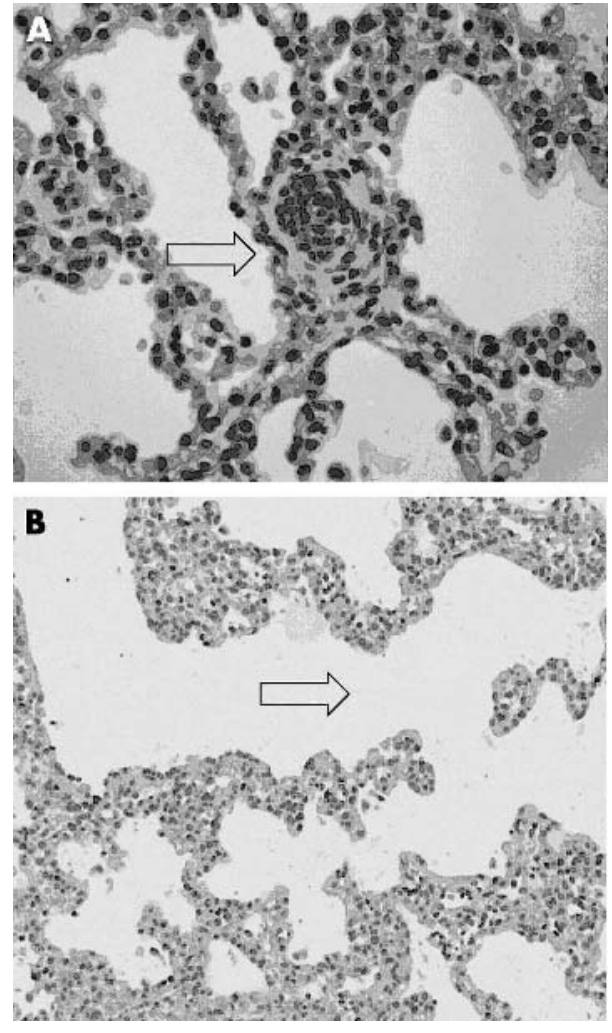

Figure 1 Lung histology. (A) Arrow showing pulmonary vasculopathy with intimal proliferation and marked medial thickening. (B) Arrow showing impaired alveolarisation.

stayed in the intensive care unit for a total of 7 days and in the hospital for a total of 31 days.

At hospital discharge the patient was acyanotic with no respiratory distress. Persistent RVH was seen on the ECG and echocardiogram, and the TR jet was $4.0 \mathrm{~m} / \mathrm{s}$. Her medications included sildenafil $4.0 \mathrm{mg}$ every $4 \mathrm{~h}$, frusemide $3.0 \mathrm{mg}$ twice a day, aspirin $20 \mathrm{mg}$ once a day, and digoxin $20 \mu \mathrm{g}$ twice a day, orally.

Follow up with serial ECGs and echocardiogram revealed gradual regression of RVH accompanied by improvement of RV function and normalisation of pulmonary artery pressures over a 7 month period. At the age of 1 year the patient no longer takes sildenafil and has no cardiac symptoms, and her cardiovascular examination, ECG, and echocardiogram are normal. No complications related to long term sildenafil therapy have been noted.

\section{DISCUSSION}

Sildenafil produces pulmonary vasodilatation by selective inhibition of phosphodiesterase type 5, responsible for degradation of cyclic-guanosine 5 monophosphate (cGMP), the second messenger of endogenous NO. Recent studies have documented the role of sildenafil in an animal model of PPHN. $^{4}$

Schulze-Neick et al have shown that in children with postoperative pulmonary hypertension sildenafil potentiates the release of CGMP to a significantly greater degree than inhaled NO for similar effect. ${ }^{5}$ Sildenafil also induces vasodilatation in poorly ventilated areas of the lung such that it can cause a drop in arterial $\mathrm{PaO}_{2}$ due to intrapulmonary shunting. Thus its more marked effect on CGMP and its widespread effect on the pulmonary vasculature may explain the superiority of sildenafil in this case.

The apparent long term resolution of pulmonary hypertension could be due to adequate alveolar growth and lung maturation in the early postnatal period accompanied by regression of pulmonary arterial medial hypertrophy with the formation of new peripheral vessels. Inhaled NO was shown to improve alveolarisation and lung growth in an animal model $^{6}$ and sildenafil may have had a beneficial effect on postnatal alveolar growth by increasing the bioavailability of cGMP. However, the long term outcome without the suppressive effect of sildenafil and the response to further insults in later life remain to be determined in this patient.

\section{CONCLUSION}

The role of sildenafil in neonatal pulmonary hypertension needs to be evaluated. This case report demonstrates its beneficial pulmonary vasodilator effect in neonates with severe pulmonary hypertension when conventional treatment fails or is contraindicated.

\section{ACKNOWLEDGEMENTS}

We thank Mr Hasan, Consultant Paediatric Cardiothoracic Surgeon for performing a lung biopsy on this patient.

\section{Authors' affiliations}

M Chaudhari, M Vogel, Department of Paediatric Cardiology, Freeman Hospital, Newcastle upon Tyne, UK

C Wright, Department of Pathology, Royal Victoria Infirmary, Newcastle upon Tyne, UK

J Smith, Paediatric Anaesthesiology, Freeman Hospital, Newcastle upon Tyne, UK

S G Haworth, Institute of Child Health, Great Ormond Street Hospital, London, UK

Competing interests: none declared

Consent was obtained for the publication of these patient details

Correspondence to: Milind Chaudhari, Department of Paediatric Cardiology, Freeman Hospital, Newcastle upon Tyne, NE7 7DN, UK; milind.chaudhari@nuth.northy.nhs.uk

Accepted 1 February 2005

\section{REFERENCES}

1 Reid LM. Pre- and postnatal development of pulmonary circulation. In: Chernick V, Mullins RB, eds. Basic mechanisms of pediatric respiratory disease: cellular and integrative. Philadelphia, PA: BC Decker, 1991:36-54

2 Lassus $P$, Turanlahti $M$, Heikkila $P$, et al. Pulmonary vascular endothelial growth factor and Flt- 1 in fetuses, in acute and chronic lung disease, and in persistent pulmonary hypertension of the newborn. Am J Respir Crit Care Med 2001;164:1981-7.

3 Schreiber MD, Gin-Mestan K, Marks J D, et al. Inhaled nitric oxide in premature infants with the respiratory distress syndrome. N Engl J Med 2003;349:2099-107.

4 Shekerdemian LS, Ravn HB, Penny DJ. Intravenous Sildenafil lowers pulmonary vascular resistance in a model of neonatal pulmonary hypertension. Am J Respir Crit Care Med 2002;165:1098-102.

5 Schulze-Neick I, Hartenstein P, Stiller B, et al. Intravenous Sildenafil is a potent pulmonary vasodilator in children with congenital heart disease. Circulation 2003; 108:11167-73

6 Tang JR, Markham NE, Lin YJ, et al. Inhaled nitric oxide attenuates pulmonary hypertension and improves lung growth in infant rats after neonatal treatment with a VEGF receptor inhibitor. Am J Physiol Lung Cell Mol Physiol 2004;287:L344-L351. 